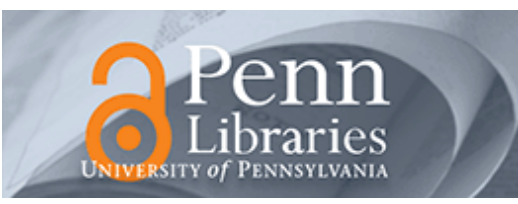

University of Pennsylvania

ScholarlyCommons

\title{
Angle-Resolved Environmental X-Ray Photoelectron Spectroscopy: A New Laboratory Setup for Photoemission Studies at Pressures up to 0.4 Torr
}

\author{
F. Mangolini \\ University of Pennsylvania \\ J. Åhlund \\ VG Scientia $A B$ \\ Graham E. Wabiszewski \\ University of Pennsylvania, grahamw@seas.upenn.edu \\ Vivek P. Adiga \\ University of Pennsylvania, adiga@seas.upenn.edu \\ Follow this and additional works at: https://repository.upenn.edu/meam_papers \\ D Fgberts

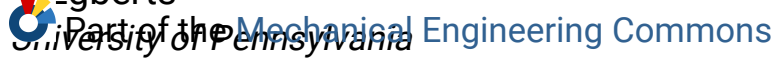

\section{Becquxmeagdefo, Citatitional authors}

Mangolini, F.; Åhlund, J.; Wabiszewski, Graham E.; Adiga, Vivek P.; Egberts, P.; Streller, F.; Backlund, K.;

Karlsson, P. G.; Wannberg, B.; and Carpick, Robert W., "Angle-Resolved Environmental X-Ray Photoelectron Spectroscopy: A New Laboratory Setup for Photoemission Studies at Pressures up to 0.4 Torr" (2012).

Departmental Papers (MEAM). 296.

https://repository.upenn.edu/meam_papers/296

F. Mangolini, J. Åhlund, G. E. Wabiszewski, V. P. Adiga, P. Egberts, F. Streller, K. Backlund, P. G. Karlsson, B. Wannberg, and R. W. Carpick. (2012). Angle-resolved environmental X-ray photoelectron spectroscopy: A new laboratory setup for photoemission studies at pressures up to 0.4 Torr. Review of Scientific Instruments, 83(9), 093112. doi: 10.1063/1.4754127

Copyright 2012 American Institute of Physics. This article may be downloaded for personal use only. Any other use requires prior permission of the author and the American Institute of Physics.

This paper is posted at ScholarlyCommons. https://repository.upenn.edu/meam_papers/296

For more information, please contact repository@pobox.upenn.edu. 


\title{
Angle-Resolved Environmental X-Ray Photoelectron Spectroscopy: A New Laboratory Setup for Photoemission Studies at Pressures up to 0.4 Torr
}

\author{
Abstract \\ The paper presents the development and demonstrates the capabilities of a new laboratory-based \\ environmental X-ray photoelectron spectroscopy system incorporating an electrostatic lens and able to \\ acquire spectra up to 0.4 Torr. The incorporation of a two-dimensional detector provides imaging \\ capabilities and allows the acquisition of angle-resolved data in parallel mode over an angular range of \\ $14^{\circ}$ without tilting the sample. The sensitivity and energy resolution of the spectrometer have been \\ investigated by analyzing a standard $\mathrm{Ag}$ foil both under high vacuum $\left(10^{-8} \mathrm{Torr}\right)$ conditions and at \\ elevated pressures of $\mathrm{N}_{2}$ ( 0.4 Torr). The possibility of acquiring angle-resolved data at different pressures \\ has been demonstrated by analyzing a silicon/silicon dioxide $\left(\mathrm{Si} / \mathrm{SiO}_{2}\right)$ sample. The collected angle- \\ resolved spectra could be effectively used for the determination of the thickness of the native silicon \\ oxide layer. \\ Disciplines \\ Mechanical Engineering

\section{Comments} \\ F. Mangolini, J. Åhlund, G. E. Wabiszewski, V. P. Adiga, P. Egberts, F. Streller, K. Backlund, P. G. Karlsson, B. \\ Wannberg, and R. W. Carpick. (2012). Angle-resolved environmental X-ray photoelectron spectroscopy: A \\ new laboratory setup for photoemission studies at pressures up to 0.4 Torr. Review of Scientific \\ Instruments, 83(9), 093112. doi: 10.1063/1.4754127
}

Copyright 2012 American Institute of Physics. This article may be downloaded for personal use only. Any other use requires prior permission of the author and the American Institute of Physics.

\section{Author(s)}

F. Mangolini, J. Åhlund, Graham E. Wabiszewski, Vivek P. Adiga, P. Egberts, F. Streller, K. Backlund, P. G. Karlsson, B. Wannberg, and Robert W. Carpick 


\title{
Angle-resolved environmental X-ray photoelectron spectroscopy: A new laboratory setup for photoemission studies at pressures up to 0.4 Torr
}

\author{
F. Mangolini, ${ }^{1}$ J. Åhlund, ${ }^{2}$ G. E. Wabiszewski, ${ }^{1}$ V. P. Adiga, ${ }^{3, \text { a) }}$ P. Egberts, ${ }^{1}$ F. Streller, ${ }^{3}$ \\ K. Backlund, ${ }^{2}$ P. G. Karlsson, ${ }^{2}$ B. Wannberg, ${ }^{2,4}$ and R. W. Carpick ${ }^{1,3, b)}$ \\ ${ }^{1}$ Department of Mechanical Engineering and Applied Mechanics, University of Pennsylvania, \\ 220 S. 33rd Street, Philadelphia, Pennsylvania 19104, USA \\ ${ }^{2}$ VG Scienta AB, Box 15120, SE-750 15 Uppsala, Sweden \\ ${ }^{3}$ Department of Materials Science and Engineering, University of Pennsylvania, 3231 Walnut Street, \\ Philadelphia, Pennsylvania 19104, USA \\ ${ }^{4}$ BW Particle Optics AB, P.O. Box 55, SE-822 22 Alfta, Sweden
}

(Received 2 July 2012; accepted 6 September 2012; published online 27 September 2012)

\begin{abstract}
The paper presents the development and demonstrates the capabilities of a new laboratory-based environmental X-ray photoelectron spectroscopy system incorporating an electrostatic lens and able to acquire spectra up to 0.4 Torr. The incorporation of a two-dimensional detector provides imaging capabilities and allows the acquisition of angle-resolved data in parallel mode over an angular range of $14^{\circ}$ without tilting the sample. The sensitivity and energy resolution of the spectrometer have been investigated by analyzing a standard $\mathrm{Ag}$ foil both under high vacuum $\left(10^{-8}\right.$ Torr $)$ conditions and at elevated pressures of $\mathrm{N}_{2}(0.4$ Torr). The possibility of acquiring angle-resolved data at different pressures has been demonstrated by analyzing a silicon/silicon dioxide $\left(\mathrm{Si} / \mathrm{SiO}_{2}\right)$ sample. The collected angle-resolved spectra could be effectively used for the determination of the thickness of the native silicon oxide layer. () 2012 American Institute of Physics. [http://dx.doi.org/10.1063/1.4754127]
\end{abstract}

\section{INTRODUCTION}

Among all the weapons in the surface-analysis arsenal, $\mathrm{X}$-ray photoelectron spectroscopy (XPS) is one of the most powerful. ${ }^{1}$ Besides providing valuable information about the surface chemistry of both conductive and insulating samples, XPS can be effectively used for chemical state, elemental, and valence band mapping, destructive and non-destructive depth-profiling, acquiring spin-resolved spectra, performing diffraction studies, and determining the thickness of thin films with sub-nanometer resolution. ${ }^{1,2}$ The wealth of detailed information XPS yields derives from both the technical progress made by manufacturers of photoelectron spectrometers and from the development of new methods, standards of operation and quantification procedures made by researchers in the last decades. ${ }^{1,2}$

Because of the relatively short mean free path of electrons with kinetic energies below $1500 \mathrm{eV}$ in a gas at elevated pressures, as well as the vacuum requirements for both X-ray anodes and electron multipliers, XPS measurements are conventionally performed under high vacuum or ultrahigh vacuum (UHV) conditions. ${ }^{3}$ However, at room temperature, a sizable change in surface free energy occurs between UHV and atmospheric pressures, which might result in changes in surface chemistry and stability. ${ }^{4}$ This creates a "pressure gap," which for years has not only hindered the use of XPS for studying surfaces (either of solids or liquids) in equilibrium with a gas or vapor, but has also prevented the use of XPS for carrying out fundamental studies of cataly-

a) Present address: School of Applied and Engineering Physics, Cornell University, Ithaca, New York 14853, USA.

b) Author to whom correspondence should be addressed. Electronic mail: carpick@seas.upenn.edu. sis and environmental science, where information about surfaces exposed to gases or liquids at different pressures is highly desirable. ${ }^{4-10}$ To overcome this issue, environmental XPS (E-XPS) has been developed ${ }^{4-10}$ (note: in the present paper the term "environmental XPS" has been selected instead of "ambient-pressure XPS" and "high-pressure XPS" since the instrument presented here, as well as other XPS instrumentation up to date, ${ }^{4-10}$ is not capable of performing spectroscopic measurements at pressures as high as $1 \mathrm{~atm}$ or higher. Since the working principle of these photoelectron spectrometers is similar to that of other instrumentation widely used in scientific research such as "environmental scanning electron microscopy," the term "environmental XPS" is used throughout the paper as it is consistent with other literature). The basic principle of this method is to reduce the attenuation of the electrons due to scattering by gas molecules by minimizing their path length in the elevated-pressure region. This has been achieved by developing spectrometers with differentially pumped stages: the analysis chamber is separated from the analyzer optics and the X-ray source by means of, respectively, small apertures and windows.

This approach to designing E-XPS systems began in the late 1960s, when Siegbahn and his colleagues carried out gasphase experiments and investigated liquid/vapor interfaces up to approximately 0.1 Torr. ${ }^{11}$ Since then, improvements in the design of E-XPS systems have allowed measurements with progressively higher sensitivity to be performed. ${ }^{8}$ The latest generation of E-XPS spectrometers incorporates a differential pumping system in combination with an electrostatic lens, allowing the acquisition of XPS spectra up to approximately 25 Torr with minimal photoelectron intensity losses. ${ }^{4-8,10,12}$

Many of the E-XPS systems described in the literature and currently in use have been developed for operation 
at synchrotron light sources. Although synchrotron radiation provides a focused light source with high brightness and intensity, the availability of beamline time at synchrotron facilities limits the throughput of these newly developed systems.

Therefore, laboratory-based E-XPS systems have been developed. ${ }^{12-14}$ This paper presents the development and demonstrates the capabilities of a laboratory-based environmental XPS system incorporating an electrostatic lens system, which is able to acquire spectra up to 0.4 Torr. The incorporation of a two-dimensional (2D) detector provides imaging capabilities and allows the acquisition of angleresolved data in parallel mode over an angular range of $14^{\circ}$. The capabilities of the spectrometer are demonstrated by analysis of a standard $\mathrm{Ag}$ foil at various pressures and a $\mathrm{Si} / \mathrm{SiO}_{\mathrm{x}}$ sample in angle-resolved mode both under high vacuum $\left(10^{-8}\right.$ Torr $)$ conditions and at elevated pressure (0.1 Torr). No laboratory-based E-XPS system with similar angular resolving capabilities has been reported before in the literature.

\section{DESIGN OF THE SPECTROMETER}

The XPS system described here is one of the key components of an elaborate surface science apparatus developed in our laboratory. Besides including an X-ray source and electron energy analyzer (VG Scienta AB, Uppsala, Sweden), the XPS chamber (VG Scienta AB, Uppsala, Sweden) includes several additional components: a differentially pumped ion gun (model: PHI 04-303, Perkin-Elmer Physical Electronics, $\mathrm{MN}$ ), a low-energy electron diffraction-Auger optics system (model: BDL600IR, Vacuum Microengineering, Canada), a mass spectrometer for residual gas analysis (model: RGA200, Stanford Research Systems, CA), and a variable temperature (80-1200 K) sample stage (VG Scienta Ltd., St. Leonards-onSea, UK), which can be used to rotate the sample about two orthogonal axes. Leaking different gases into the XPS analysis chamber is achieved by means of a standard leak valve. The pumping system of the XPS analysis chamber includes a turbomolecular pump (model: TMP 361, pumping speed for $\mathrm{N}_{2}: 345 \mathrm{l} / \mathrm{s}$, Oerlikon Leybold Vacuum GmbH, Germany) and an ion/titanium sublimation pump (TiTan ${ }^{\mathrm{TM}} 300 \mathrm{~T}$ Ion Pump, pumping speed for $\mathrm{N}_{2}: 300$ 1/s, Gamma Vacuum, MN).

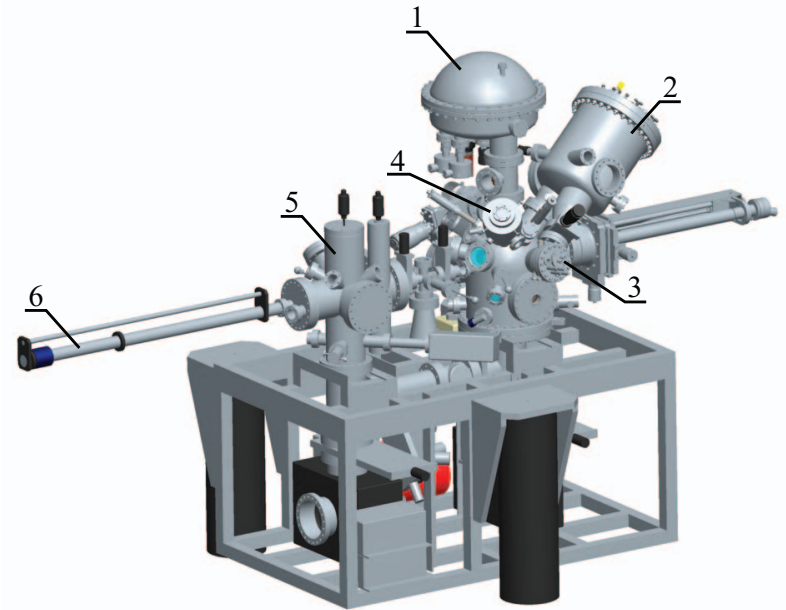

FIG. 1. Three-dimensional computer-aided design model of the surface science apparatus (the turbomolecular pumps are excluded for clarity): (1) electron energy analyzer; (2) X-ray monochromator; (3) X-ray source; (4) differentially pumped ion gun; (5) UHV scanning probe microscopy chamber; (6) magnetic linear transfer probe.

The chamber is extensively lined internally with mu-metal shielding. The XPS chamber is coupled to a second chamber, where a high-resolution, variable temperature (35-700 K) ultra-high vacuum scanning probe microscope (SPM) (RHK $750 \mathrm{AFM} / \mathrm{STM}$, RHK Technology) is located. The SPM apparatus can operate in all atomic force microscopy (AFM) and scanning tunneling microscopy modes, including noncontact (i.e., frequency modulation) AFM using the phaselocked loop technique. Multiple samples can be stored in the SPM chamber, which is pumped by both a turbomolecular pump (model: HiPace 400, pumping speed for $\mathrm{N}_{2}: 355 \mathrm{l} / \mathrm{s}$, Pfeiffer Vacuum GmbH, Germany) and an ion pump/titanium sublimation pump (TiTan ${ }^{\mathrm{TM}} 300 \mathrm{~T}$ Ion Pump, pumping speed for $\mathrm{N}_{2}: 300$ 1/s, Gamma Vacuum, MN). Sample introduction into the surface science apparatus is achieved through a fast load-lock by means of a magnetic linear transfer probe. A 3D computer-aided design model of the system is displayed in Figure 1.

In Secs. II A and II B, the design of the two main parts of the new environmental X-ray photoelectron spectrometer, namely, the X-ray source and the electron energy analyzer, will be presented.
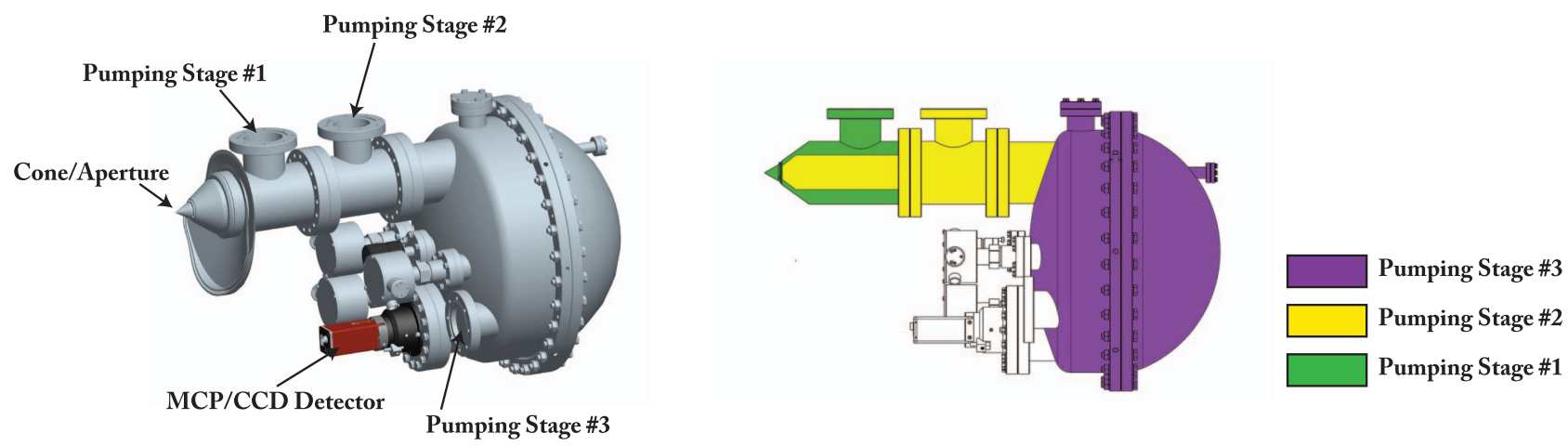

FIG. 2. Schematic diagrams of the Scienta R3000 environmental electron energy analyzer. 


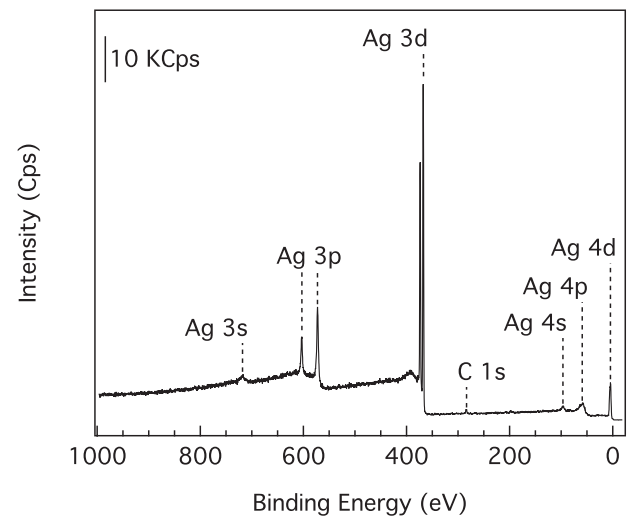

FIG. 3. Survey spectrum of a Ag foil acquired at $1.0 \times 10^{-8}$ Torr. Acquisition parameters: pass energy: $200 \mathrm{eV}$; slit: $3.0 \mathrm{~mm}$ straight; step size: $300 \mathrm{meV}$; dwell time: $0.133 \mathrm{~s}$; acquisition time: $27 \mathrm{~min}$.

\section{A. X-ray source}

The X-ray source is a customized version of the Scienta MX 650 X-ray source. It consists of an X-ray anode (Scienta SAX 100) and an X-ray monochromator (Scienta XM 780). X-rays are generated by bombarding a water-cooled aluminum anode with a finely focused electron beam originating from a tungsten cathode. Under normal working conditions, the acceleration voltage and emission current are $15 \mathrm{kV}$ and $30 \mathrm{~mA}$, respectively. The $\mathrm{X}$-ray anode is separated from the $\mathrm{X}$-ray monochromator by means of a gate valve connected to an interlock, which protects the anode in case of an unexpected pressure increase.

The X-ray monochromator consists of seven toroidally bent $\alpha$-quartz crystals each having a diameter of $76 \mathrm{~mm}$. The crystals are kept at elevated temperature ( $328 \mathrm{~K}$ ) by two quartz light bulbs to ensure that the quartz crystal lattice spacing is not affected by temperature variations in the laboratory environment. They are arranged in a close-packed array on a Rowland circle having a diameter of $650 \mathrm{~mm}$. The dispersion and focus of each crystal can be individually adjusted by means of screws present on the lid of the monochro- mator where the crystals are located. The monochromatized $\mathrm{X}$-rays have an energy of $1486.6 \mathrm{eV}$ with an energy width of $168 \mathrm{meV}^{15}$ The X-ray beam has the same dimensions as the electron beam impinging onto the aluminum anode, i.e., $1 \times 3 \mathrm{~mm}^{2}$. The X-ray monochromator is pumped by a turbomolecular pump (model TMU 520, pumping speed for $\mathrm{N}_{2}$ : 520 1/s, Pfeiffer Vacuum GmbH, Germany).

A reinforced aluminum foil (thickness: $200 \mathrm{~nm}$ ) separates the X-ray monochromator from the XPS analysis chamber. This foil is designed to withstand pressure differences of at least 10 Torr. The distance between the X-ray window and the analyzer focal point is $102 \mathrm{~mm}$. The center of the $\mathrm{X}$-ray monochromator is situated in the plane defined by the lens axis and the entrance slit of the hemispherical deflector. The angle between the $\mathrm{X}$-ray source and the lens axis is $50^{\circ}$.

\section{B. Analyzer design}

The environmental XPS electron energy analyzer is based on the Scienta R3000 analyzer series. It consists of an electrostatic lens system and a concentric hemispherical analyzer (CHA) with a mu-metal tank and an additional mu-metal liner for magnetic shielding.

The electron energy analyzer can operate in both transmission mode and angular mode: ${ }^{16,17}$ in the former, the elements of the electrostatic lens are set to maximize the signal intensity while preserving spatial information. This mode of operation is the standard mode of acquiring XPS spectra thanks to the possibility of collecting data with high signal-tonoise ratio from both small and large X-ray spots. In angular mode, the elements of the electrostatic lens system are set so that photoelectrons emitted at different emission angles (the emission angle is the angle between the surface normal and the direction of the photoemitted electrons ${ }^{18}$ ) are refocused onto different position of the detector.

A differentially pumped electrostatic lens has been developed to allow XPS measurements at elevated pressures to be performed while maintaining the multichannel plate (MCP) (a)

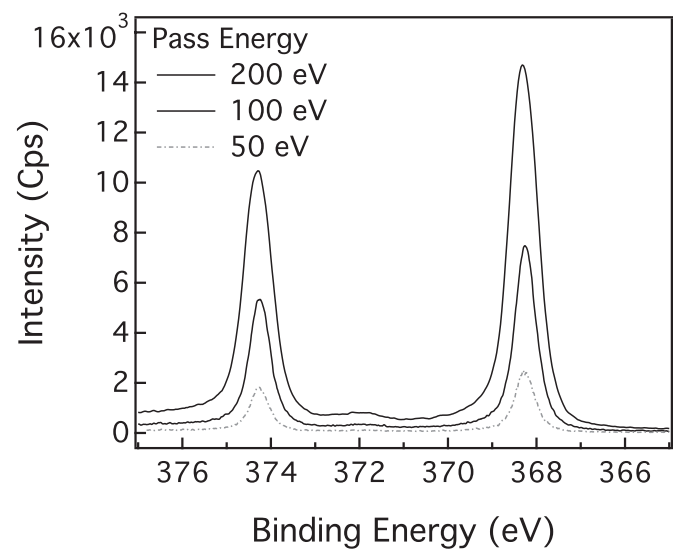

(b)

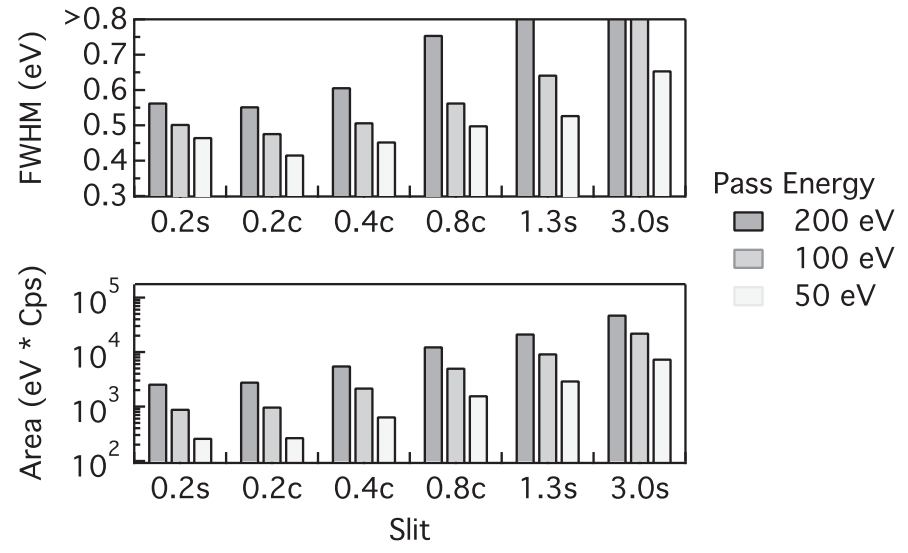

FIG. 4. (a) High resolution XPS spectra (Ag $3 d$ ) of a scraped Ag foil acquired at $1.0 \times 10^{-8}$ Torr using different pass energies (200, 100, and $\left.50 \mathrm{eV}\right)$. Acquisition parameters: slit: $0.8 \mathrm{~mm}$ curved; step size: $15 \mathrm{meV}$; dwell time: $0.267 \mathrm{~s}$; acquisition time per spectrum: $6 \mathrm{~min}$; (b) Ag $3 d_{5 / 2}$ full width at half-maximum (FWHM) and area for different acquisition settings (pass energy and slit) in the case of the spectra acquired at $1.0 \times 10^{-8}$ Torr. The labels on the $\mathrm{x}$-axis include both the slit length in mm (numbers) and geometry ( $s$ stands for "straight" and $c$ stands for "curved"). 
detector at pressures below the Corona discharge region (ca. $1.0 \times 10^{-4}$ Torr). The lens consists of three pumping zones separated from each other by means of apertures (Figure 2). The first two apertures (cones) are changeable. For the experiments presented in this paper, the first aperture, which separates the first pumping zone from the analysis chamber that can be at elevated pressures, has a diameter of $1.2 \mathrm{~mm}$, whereas the second aperture, which separates the first and second pumping zones, has a diameter of $8 \mathrm{~mm}$. In the second pumping zone, the photoelectrons are focused onto the analyzer entrance slit, for which a number of widths and shapes are available (from 0.2 to $3.0 \mathrm{~mm}$, where the slit can be either straight or curved. A straight slit gives a curved image on the $2 \mathrm{D}$ detector, whereas a curved slit gives a straight image on the 2D detector. As a result of this, spectra acquired using straight slits are broader than those collected with curved slits). The third pumping zone is the CHA itself, which has a central radius of $135 \mathrm{~mm}$ and operates in constant analyzer energy mode. Differential pumping is achieved by means of turbomolecular pumps: while the first two stages are pumped by turbopumps having a pumping speed for $\mathrm{N}_{2}$ of $230 \mathrm{l} / \mathrm{s}$ (model TMU 261 P, Pfeiffer Vacuum GmbH, Germany), the electron energy analyzer is pumped by means of a turbopump with a pumping speed for $\mathrm{N}_{2}$ of $60 \mathrm{l} / \mathrm{s}$ (model TMU $071 \mathrm{P}$, Pfeiffer Vacuum $\mathrm{GmbH}$, Germany). Although the maximum collection angle of the Scienta R3000 analyzer is $30^{\circ}$ in transmission mode and $20^{\circ}$ in angular mode, the presence of apertures in the analyzer system limits the range, over which XPS spectra can be acquired, to $14^{\circ}$.

Electron ray-tracing calculations (see the Appendix for details) were performed to investigate the influence of the front two apertures on: (a) the area on the sample surface from which the electrostatic lens collects photoelectrons; and (b) the acceptance angle of the lens system. In transmission mode, the analyzed area is determined by the first aperture and was found to be approximately $1.2 \mathrm{~mm}$ in diameter. In the case of angle-resolved XPS (AR-XPS) measurements, the acceptance angle of the electrostatic lens is limited by the second aperture whose diameter is $8 \mathrm{~mm}$. A set of angular lens tables is also available in the analyzer software and can be used to limit the angular range over which photoelectrons are collected to 6 or $10^{\circ}$, i.e., below the maximum acceptance angle of the electrostatic lens $\left(14^{\circ}\right)$.

The electron detector consists of a 2D MCP, which is monitored by a FireWire CCD camera. The MCP has 800 channels available in each direction. The incorporation of a 2D detector opens the possibility of either acquiring angleresolved XPS data in parallel mode (i.e., without tilting the sample) or to use the non-dispersive plane of the hemispherical analyzer for retaining positional information. In the former case, an energy-vs.-emission angle image is obtained, whereas in the latter, an energy-vs.-position (along a line) image is generated.

The electron energy analyzer system allows the collection of XPS spectra in both fixed and scanned mode. In fixed mode, the entire energy range required during the acquisition is dispersed over the detector, which implies that the settings of the analyzer/lens system remain constant throughout the analysis. In scanned mode, the energy settings of the analyzer/lens system change during the acquisition so that each detector channel collects electrons from the entire kinetic energy range. ${ }^{16,17}$

\section{SYSTEM SPECIFICATIONS}

In Secs. III A and III B, the sensitivity and energy resolution of the spectrometer under high vacuum conditions and at elevated pressures will be discussed together with the

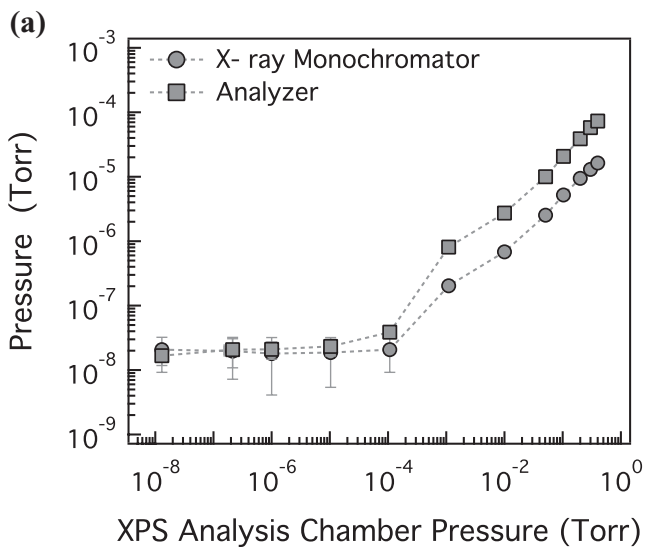

(b)

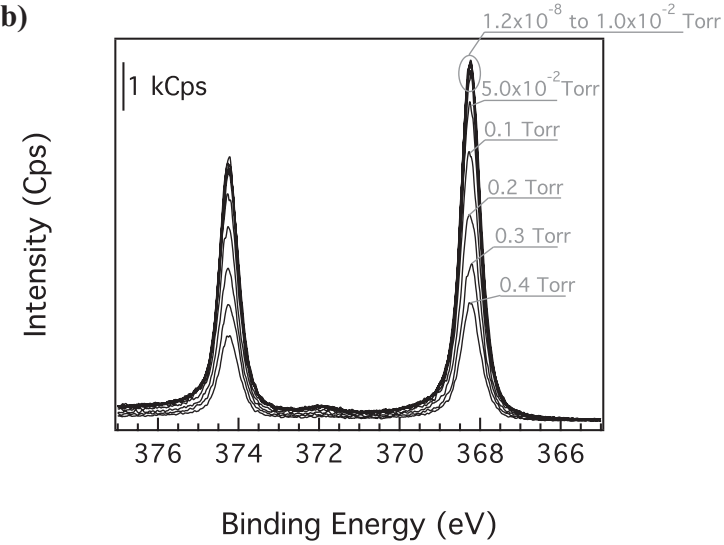

(c)

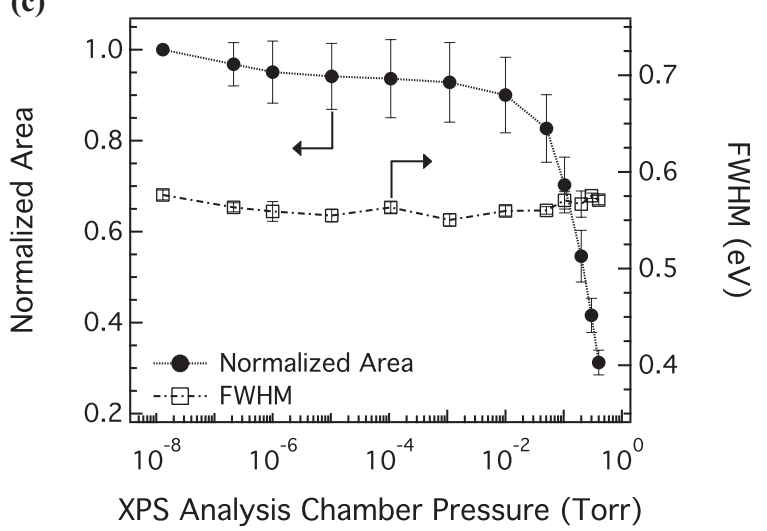

FIG. 5. (a) Pressure in the X-ray monochromator and electron energy analyzer as a function of the pressure in the XPS analysis chamber of $\mathrm{N}_{2}$ gas; (b) high resolution $\mathrm{Ag} 3 d$ XPS spectra of scraped $\mathrm{Ag}$ foil acquired at different pressures of $\mathrm{N}_{2}$ (ranging from $1.2 \times 10^{-8}$ to 0.4 Torr) in the XPS analysis chamber. Acquisition parameters: pass energy: $100 \mathrm{eV}$; slit: $0.8 \mathrm{~mm}$ curved; step size: $15 \mathrm{meV}$; dwell time: $0.267 \mathrm{~s}$; acquisition time per spectrum: $6 \mathrm{~min}$; (c) $\mathrm{Ag} 3 d_{5 / 2}$ normalized area and full width at half-maximum (FWHM) as a function of the pressure in the XPS analysis chamber. 
performance of the differentially pumped system. In Sec. III $\mathrm{C}$, the ability to acquire angle-resolved XPS data in parallel mode both under high vacuum conditions and at elevated pressure will be demonstrated.

All the spectra reported in the following were processed using CasaXPS software (v2.3.16, Casa Software Ltd., Wilmslow, Cheshire, UK).

\section{A. Spectrometer sensitivity and energy resolution in high vacuum}

Following the recommendation of ISO 15470/2004, ${ }^{19}$ the sensitivity and energy resolution of the spectrometer were specified by analyzing a standard silver foil. The silver foil was scraped with a scalpel just before being introduced into the spectrometer load-lock.

Figure 3 displays the survey spectrum of the scraped silver foil acquired at $1.0 \times 10^{-8}$ Torr. All the characteristic signals of silver were detected: ${ }^{20} \mathrm{Ag} 3 s$ at $717 \mathrm{eV}, \mathrm{Ag} 3 p_{1 / 2}$ at $602 \mathrm{eV}, \mathrm{Ag} 3 p_{3 / 2}$ at $572 \mathrm{eV}, \mathrm{Ag} 3 d_{3 / 2}$ at $373 \mathrm{eV}, \mathrm{Ag} 3 d_{5 / 2}$ at $368 \mathrm{eV}, \mathrm{Ag} 4 s$ at $95 \mathrm{eV}, \mathrm{Ag} 4 p$ at $57 \mathrm{eV}$, and $\mathrm{Ag} 4 d$ at $4 \mathrm{eV}$. Besides silver, a weak $\mathrm{C} 1 s$ peak at $285 \mathrm{eV}$ was detected. The intensity of the $\mathrm{C} 1 s$ peak was lower than $5 \%$ of the $\mathrm{Ag} 3 d_{5 / 2}$ signal, as required in the ISO $15470 / 2004$ norm. ${ }^{19}$ No O $1 s$ signal has ever been detected.

The high-resolution Ag $3 d$ XP-signals acquired at $1.0 \times 10^{-8}$ Torr using different pass energies and a $0.8 \mathrm{~mm}$ curved slit are shown in Figure 4(a). The binding-energy scale was referenced to the spectrometer vacuum level. The full width at half-maximum (FWHM) and area of the $\mathrm{Ag} 3 d_{5 / 2}$, determined after the subtraction of an iterated Shirley-Sherwood background, are reported in Figure 4(b). Three independent measurements were performed to check the reproducibility of the results: the FWHM values were within the experimental uncertainty (equal to the step size, i.e., $15 \mathrm{meV}$ ), while the uncertainty of the peak area was $4 \%$.

As expected, increasing the pass energy increased the sensitivity and decreased the energy resolution of the spectrometer for all slits. The same effect (i.e., higher sensitivity and lower energy resolution) was obtained upon increasing the dimension of the slit at the entrance of the analyzer. The best energy resolution, achieved with pass energy equal to $50 \mathrm{eV}$ and a $0.2 \mathrm{~mm}$ curved slit, was found to be $0.42 \pm 0.02 \mathrm{eV}$. This experimental value is close to the theoretical energy resolution ( $408 \mathrm{meV})$, calculated using the empirical expression given by Olivero and Longbothum ${ }^{21}$ as the convolution of the natural width of the core hole (equal to $310 \mathrm{meV}$ in the case of the $\mathrm{Ag} 3 d_{5 / 2}$ transition $^{22}$ ), the width of the photon energy (equal to $168 \mathrm{meV}$ in the case of the MX650 source ${ }^{15}$ ), the temperature broadening (equal to $90 \mathrm{meV}$ for measurements at room temperature $\left.{ }^{23,24}\right)$, and the analyzer resolution (equal to $37 \mathrm{meV}$ for measurements performed at $50 \mathrm{eV}$ pass energy and using a $0.2 \mathrm{~mm}$ curved $\mathrm{slit}^{25}$ ).

The theoretical calculation also indicates that in the case of the $\mathrm{Ag} 3 d_{5 / 2}$ transition the measured energy resolution is primarily determined by the core hole lifetime. Therefore, in the hypothetical limit of an infinitely sharp emission line, the attainable instrumental resolution, calculated as the convolution of the width of the photon energy, the temperature broadening, and the analyzer resolution, would be $194 \mathrm{meV}$.
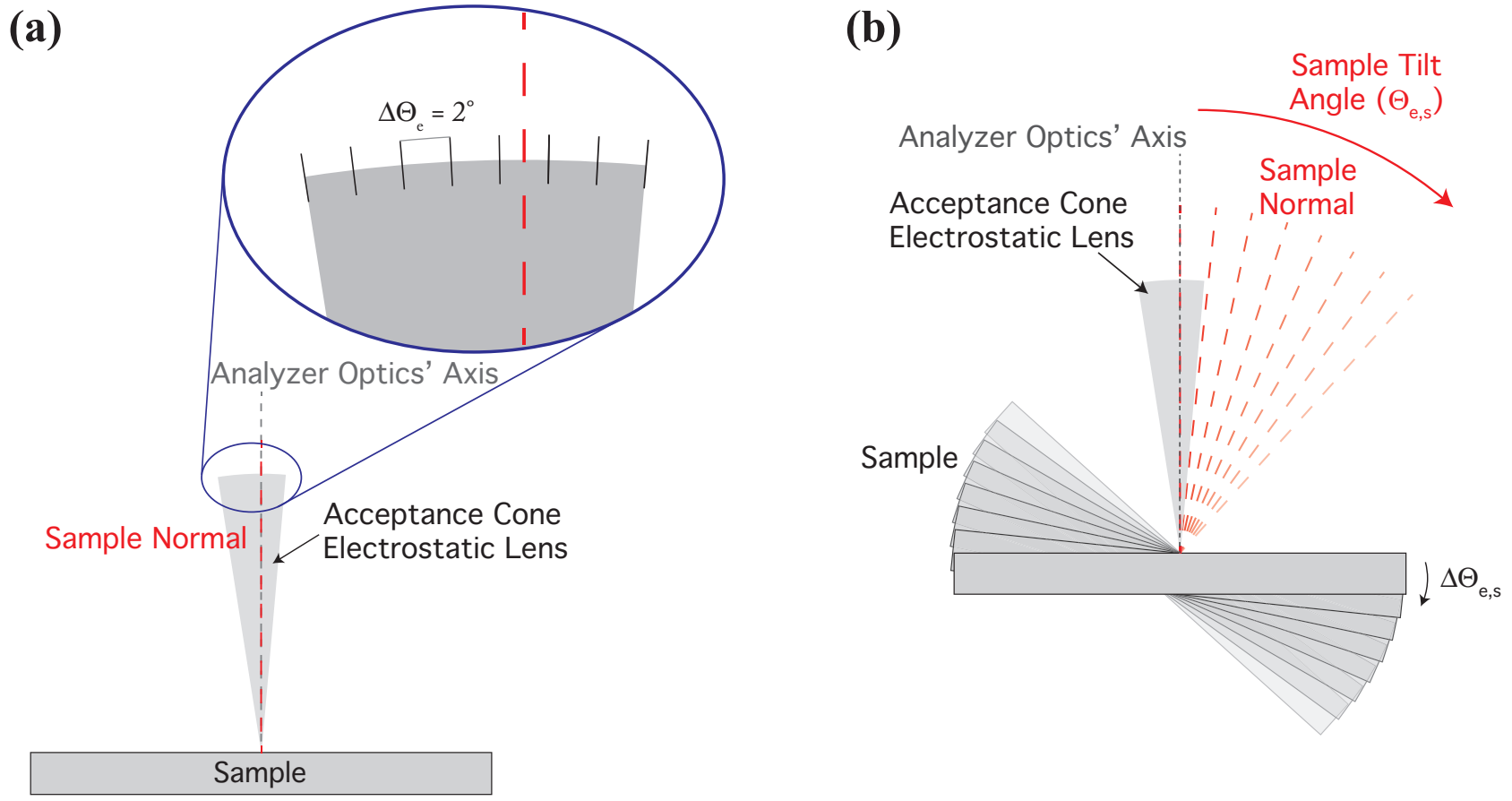

FIG. 6. (a) Acceptance angle of the electrostatic lens system. The collection cone has a $2^{\circ}$ offset with respect to the analyzer input lens axis. The collected angle-resolved XPS spectra were averaged over sectors of $2^{\circ}$ to increase the signal-to-noise ratio. (b) Experimental procedure employed for acquiring angleresolved XPS spectra: besides acquiring data in parallel mode, the sample was progressively tilted (i.e., the angle between the surface normal and the analyzer input lens $\left(\Theta_{\mathrm{e}, \mathrm{s}}\right)$ was changed $)$. 


\section{B. Spectrometer sensitivity and energy resolution at elevated pressures}

High-resolution $\mathrm{Ag} 3 d$ spectra were also acquired at pressures ranging from $1.2 \times 10^{-8}$ Torr to 0.4 Torr. The pressure in the system was adjusted by leaking $\mathrm{N}_{2}$ into the XPS analysis chamber using a standard UHV leak valve, after closing the gate valves connecting the ion and turbo pumps to the main chamber. The effectiveness of the differentially pumped system described in Sec. II was checked by monitoring the pressure in both the X-ray monochromator and the electron energy analyzer while leaking $\mathrm{N}_{2}$ into the XPS main chamber (Figure 5(a)). No significant pressure changes occurred in either the X-ray monochromator or the electron energy analyzer up to a XPS analysis chamber pressure of $1.0 \times 10^{-4}$ Torr. Raising the pressure in the XPS main chamber beyond this value resulted in a linear increase of the pressure in both X-ray monochromator and energy analyzer. The maximum working pressure in these two chambers (ca. $1.0 \times 10^{-4}$ Torr), where high voltages are applied, limits the maximum attainable pressure in the XPS main chamber to 0.4 Torr.

Figure 5(b) displays the high-resolution $\mathrm{Ag} 3 d$ spectra acquired at different pressures. The FWHM and area of the Ag $3 d_{5 / 2}$, determined after the subtraction of an iterated ShirleySherwood background, are reported in Figure 5(c). At the maximum attainable pressure in the XPS analysis chamber ( 0.4 Torr), the area of the $\mathrm{Ag} 3 d_{5 / 2}$ decreased to $31 \pm 3 \%$ of the intensity of the same peak acquired at $1.2 \times 10^{-8}$ Torr. The exponential dependence of the intensity of the photoelectron signal on the gas pressure agrees with the theoretical dependence of the attenuation of electrons travelling through a gas environment on the pressure. ${ }^{26}$ Raising the pressure from high vacuum $\left(1.2 \times 10^{-8}\right.$ Torr $)$ to elevated pressures (0.4 Torr) did not affect the spectrometer energy resolution.

\section{Angle-resolved XPS}

The recent development of parallel acquisition systems has opened the possibility of collecting data at different emission angles simultaneously (the emission angle is the angle between the surface normal and the direction of the photoemitted electrons ${ }^{18}$ ), allowing AR-XPS analysis in a non-destructive manner and without tilting the sample. ${ }^{16,27}$ Compared to conventional angle-resolved XPS, which is performed by tilting the sample to change the emission angle, acquiring XPS data in parallel mode permits the analysis of large samples, performing small-area analysis without difficulties in aligning the specimen and without any change of the analyzed area during the measurements. It also enables the characterization of insulating samples while maintaining steady charge compensation conditions. ${ }^{27}$

As already pointed out in Sec. II, the incorporation of an angle-resolving lens and a 2D detector opens the possibility of acquiring angle-resolved data in parallel mode. Although the analyzer lens has a maximum collection angle of $20^{\circ}$ in angular mode ${ }^{28}$ the presence of apertures in the lens system limits the range over which XPS spectra can be acquired to $14^{\circ}$ (see electron ray-tracing calculations reported in the Appendix). Due to a small mechanical misalignment of the first aperture

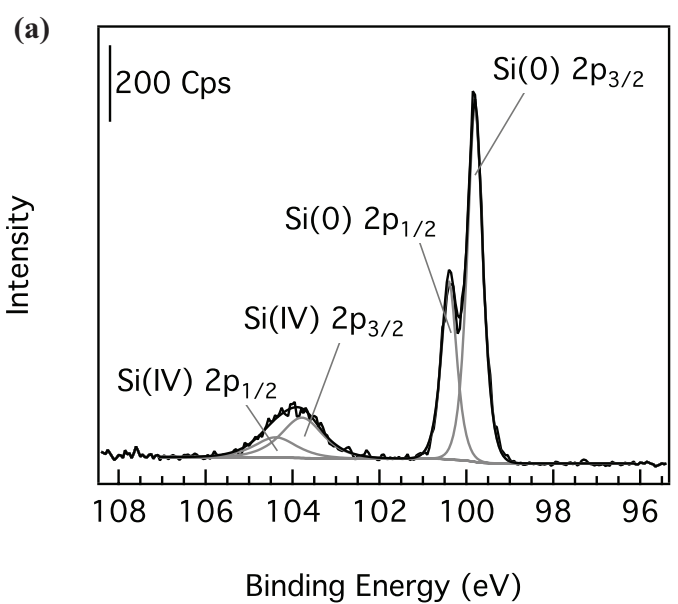

(b)

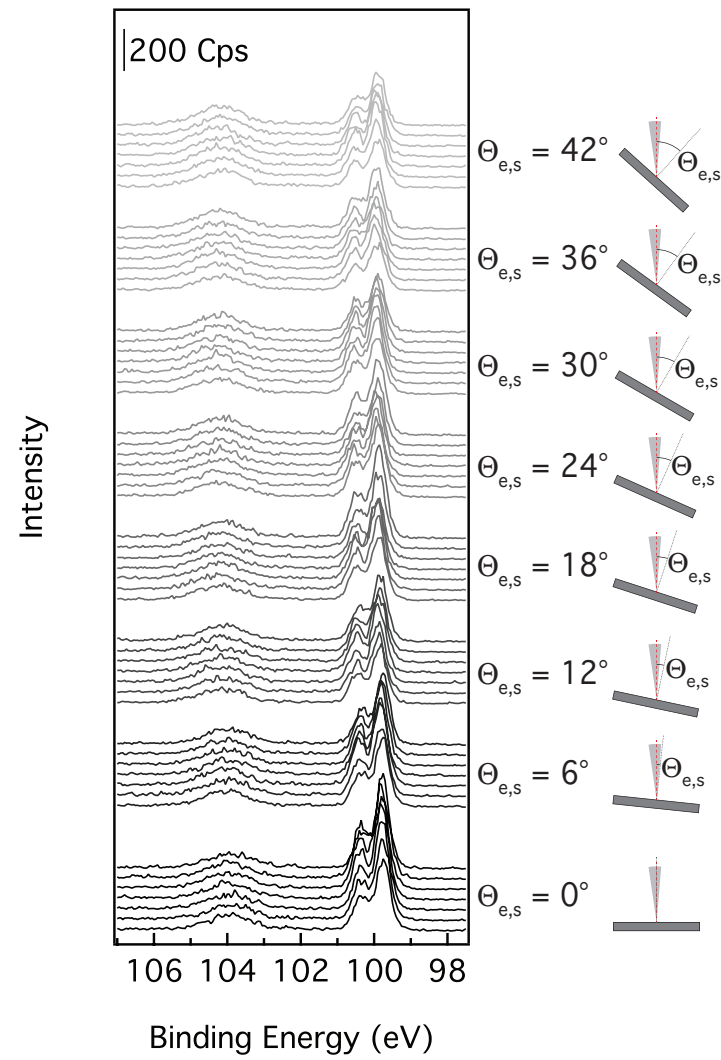

FIG. 7. (a) High-resolution Si $2 p$ XPS spectrum acquired at $1.2 \times 10^{-8}$ Torr in angle-resolved mode $\left(\Theta_{\mathrm{e}, \mathrm{s}}=0^{\circ}\right)$ of a silicon wafer with its native oxide layer. The spectrum was obtained by summing up all angular channels. Acquisition parameters: pass energy: $100 \mathrm{eV}$; slit: $0.8 \mathrm{~mm}$ curved; step size: $15 \mathrm{meV}$; dwell time: $0.267 \mathrm{~s}$; acquisition time: $55 \mathrm{~min}$; (b) XPS data were collected at $1.2 \times 10^{-8}$ Torr in parallel mode while progressively tilting the sample $\left(\Theta_{\mathrm{e}, \mathrm{s}}\right.$ from $0^{\circ}$ to $\left.42^{\circ}\right)$. The seven spectra shown at each sample tilt angle are calculated by summing over $2^{\circ}$ segments of the $14^{\circ}$ angular range.

(cone) of the electrostatic lens (which is correctable), the acceptance angle was found to have a $2^{\circ}$ offset with respect to the analyzer optics' axis (see Figure 6(a)).

In the present work, a silicon wafer with a native oxide layer (EL-CAT Inc., Waldwick, NJ) was used as-received. Data were acquired in angle-resolved mode to demonstrate the parallel acquisition capabilities of the instrument. Data acquisition in parallel angle-resolved mode was also performed while progressively tilting the sample (i.e., by changing the 
(a)

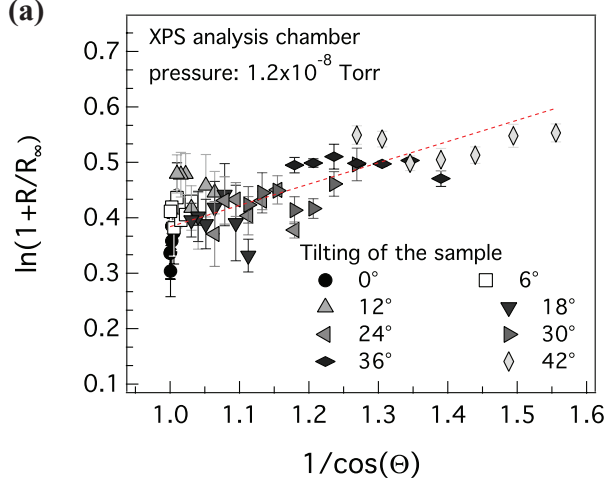

(b)

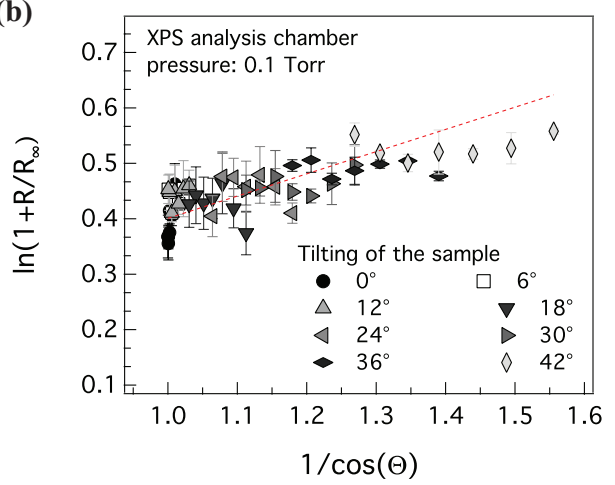

FIG. 8. Ratio of the intensities of the oxide and elemental silicon peaks $\left(\mathrm{R}=\mathrm{I}_{\mathrm{SiO}_{2}} / \mathrm{I}_{\mathrm{Si}}\right)$ as a function of the cosine of the emission angle $(\Theta)$. The angle-resolved data were collected in parallel mode while progressively tilting the sample (i.e., changing the angle between the surface normal and the analyzer lens axis) from $0^{\circ}$ to $42^{\circ}$. Since the acceptance angle of the lens system is $14^{\circ}$ and the sample was progressively tilted by $6^{\circ}$, some of the data points acquired in parallel mode but after physically tilting the sample correspond to the same emission angle. Data acquired under (a) high vacuum conditions (i.e., at $1.2 \times 10^{-8}$ Torr) and (b) at elevated pressure $(0.1$ Torr $)$

sample tilt angle $\Theta_{e, s}$, the angle between the surface normal and the analyzer input lens axis) from $0^{\circ}$ to $42^{\circ}$ in steps of $6^{\circ}$ (see Figure 6(b)). Maintaining the X-ray spot on the sample surface while tilting the specimen is facilitated by the geometry of the system, namely, the location of the center of the X-ray monochromator in the plane defined by the lens axis and the entrance slit of the hemispherical analyzer. ${ }^{29}$ The angle-resolved data acquired in parallel mode were averaged over sectors of $2^{\circ}$ to increase the signal-to-noise ratio, producing seven spectra for each sample tilt angle (see Figure 6(a)).

The survey spectrum of the silicon wafer (not shown) exhibited an intense $\mathrm{O} 1 s$ peak at $533 \mathrm{eV}$ and the characteristic signals of silicon, i.e., $\mathrm{Si} 2 p$ at $100 \mathrm{eV}$ and $\mathrm{Si} 2 s$ at $151 \mathrm{eV}^{20}$ A weak carbon peak ( $\mathrm{C} 1 s$ ) was also detected at $285 \mathrm{eV}^{20}$ In agreement with the requirements of the ISO/DIS $14701,{ }^{30}$ the intensity of the C $1 s$ peak was less than $30 \%$ of the $\mathrm{Si} 2 p$ signal intensity (larger amounts of carbonaceous contamination would lead to an increase in the uncertainty of the measured oxide thickness). The $\mathrm{C} 1 s$ signal is attributable to ambient contamination expected on the surface due to its exposure to air.

In the case of the angle-resolved high-resolution XPS spectra, a curve-fitting procedure was first performed on the spectrum obtained by summing up all the angular channels. The fitting parameters were then propagated to all angles, constraining the FWHM and position of the peaks $( \pm 0.1 \mathrm{eV})$.

The high-resolution $\mathrm{Si} 2 p$ spectrum is a convolution of the $2 p_{3 / 2}$ and $2 p_{1 / 2}$ components due to spin-orbit coupling. ${ }^{3}$ Curve synthesis was performed constraining the integrated intensity ratio of these two signals to 0.5 and their intensity separation to $0.6 .^{30}$ The $\mathrm{Si} 2 p$ signal was fitted with two doublets, whose maxima of their $2 p_{3 / 2}$ components were found at $99.7 \pm 0.1 \mathrm{eV}$ and $103.8 \pm 0.2 \mathrm{eV}$. The former is assigned to elemental silicon $(\operatorname{Si}(0))$, whereas the latter is assigned to silicon in silicon dioxide $\left(\mathrm{SiO}_{2}\right){ }^{30}$ The binding energy difference between the two signals is in agreement with the values reported in the literature. ${ }^{30,31}$ No peaks, which can be assigned to silicon with oxidation state between 0 and $+4,{ }^{30,31}$ were revealed by the curve-synthesis procedure. An example of fit- ted Si $2 p$ XP-signal is reported in Figure 7(a). The spectra acquired in parallel mode while progressively tilting the sample $\left(\Theta_{\mathrm{e}, \mathrm{s}}\right.$ from $0^{\circ}$ to $\left.42^{\circ}\right)$ are shown in Figure 7(b).

As extensively reported in the literature, ${ }^{3,27,30,31}$ calculating the ratio of the intensities of the oxide and elemental silicon peaks $\left(\mathrm{R}=\mathrm{I}_{\mathrm{SiO}_{2}} / \mathrm{I}_{\mathrm{Si}}\right)$ allows the determination of the thickness of the oxide layer $\left(\mathrm{d}_{\mathrm{SiO}_{2}}\right)$ according to the equation

$$
\mathrm{d}_{\mathrm{SiO}_{2}}=\mathrm{L}_{\mathrm{SiO}_{2}} \cos \Theta \ln \left[1+\frac{\mathrm{R}}{\mathrm{R}_{\infty}}\right],
$$

where $\mathrm{L}_{\mathrm{SiO}_{2}}$ is the attenuation length for the $\mathrm{Si} 2 p$ photoelectrons in $\mathrm{SiO}_{2}, \Theta$ is the emission angle, and $\mathrm{R}_{\infty}$ is the ratio of the intensities of the oxide and elemental silicon peaks in the case of bulk samples $\left(\mathrm{R}_{\infty}=\mathrm{I}_{\mathrm{SiO}_{2}}^{\infty} / \mathrm{I}_{\mathrm{Si}}^{\infty}\right)$.

Plotting the logarithmic function appearing in Eq. (1) as a function of the inverse of the cosine of the emission angle will produce a line whose slope is the ratio of the thickness of the oxide layer and the photoelectron attenuation length $\left(\mathrm{d}_{\mathrm{SiO}_{2}} / \mathrm{L}_{\mathrm{SiO}_{2}}\right)$. The data acquired in parallel mode and by progressively tilting the sample are displayed in this way in Figure 8. Following the recommendations of the ISO/DIS $14701,{ }^{30}$ an $R_{\infty}$ value of 0.9329 was used. Since the acceptance angle of the lens system is $14^{\circ}$ and the sample was progressively tilted by $6^{\circ}$, some of the data points acquired in parallel mode while physically tilting the sample correspond to the same emission angle. These data points were found to nicely overlap.

The experimental data shown in Figure 8 were fitted with a straight line passing through the origin. The fitting procedure was first performed considering the intensity ratios determined from the spectra acquired in parallel mode at a certain sample tilt angle (i.e., without any change in the physical tilt of the specimen). To calculate the thickness of the oxide layer, the value of $3.485 \mathrm{~nm}$ was used for the photoelectron attenuation length $\left(\mathrm{L}_{\mathrm{SiO}_{2}}\right)$, as suggested in the ISO/DIS 14701. ${ }^{30}$ Figure 9 displays the silicon oxide thickness determined from both the spectra collected under high vacuum conditions and at elevated pressure. Small variations in the calculated values were observed upon tilting the sample. The thickness of the oxide layer determined considering the whole set of data (i.e., spectra collected in parallel mode and at different sample 


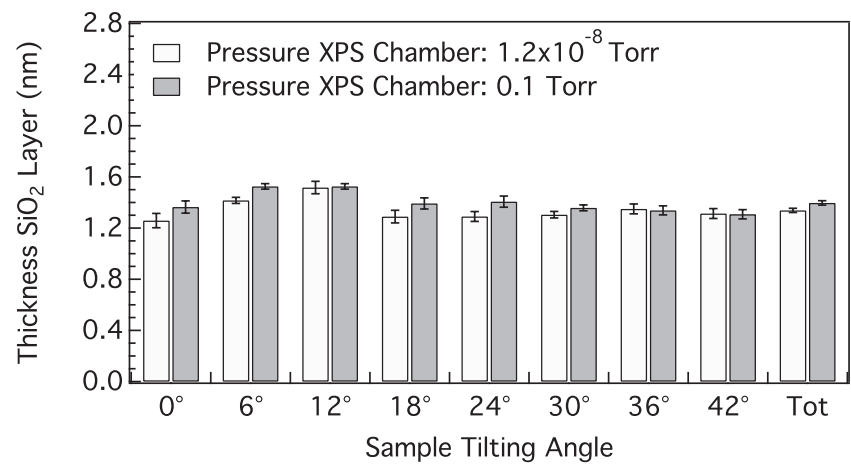

FIG. 9. Calculated thickness of the native silicon oxide layer using the XPS data acquired under high vacuum conditions $\left(1.2 \times 10^{-8}\right.$ Torr $)$ and at elevated pressure ( 0.1 Torr) shown in Figure 8. The thickness of the oxide layer was calculated considering each set of XPS spectra acquired in parallel mode at a certain sample tilt angle. The thickness of the oxide layer determined considering the whole set of data (i.e., spectra collected in parallel mode and at different sample tilt angles) is also reported.

tilting angles) was found to be $1.3_{4} \pm 0.0_{2} \mathrm{~nm}$ and $1.4_{0} \pm 0.0_{2}$ $\mathrm{nm}$ in the case of, respectively, the data acquired under high vacuum conditions $\left(1.2 \times 10^{-8}\right.$ Torr $)$ and those collected at elevated pressure ( 0.1 Torr). The calculated thickness values of the native silicon oxide layer are in agreement with those reported in the literature. ${ }^{32}$

The fit of the whole set of data, i.e., considering all the intensity ratios calculated from the spectra acquired in parallel mode while physically tilting the sample, provided thickness values comparable to the ones determined using the data collected in parallel mode without any physical change in the specimen orientation.

This finding indicates that, in spite of the limited acceptance angle of the electrostatic lens system, the XPS spectrometer can effectively be used for quantitatively determining the thickness of extremely thin layers.

\section{CONCLUSIONS AND OUTLOOK}

A new laboratory-based E-XPS system incorporating an electrostatic lens and able to acquire spectra up to 0.4 Torr has been developed. The incorporation of a $2 \mathrm{D}$ detector provides imaging capabilities and allows the acquisition of angleresolved data in parallel mode over an angle range of $14^{\circ}$. The sensitivity and energy resolution of the spectrometer have been investigated by analyzing a standard Ag foil both under high vacuum conditions and at elevated pressures. The best energy resolution under high vacuum conditions, quantified as FWHM of the $\mathrm{Ag} 3 d_{5 / 2}$ peak, was $0.42 \pm 0.02 \mathrm{eV}$, close to the theoretical energy resolution $(408 \mathrm{meV})$. This value was primarily determined by the natural width of the $\mathrm{Ag} 3 d_{5 / 2}$ core hole transition (310 meV) and is consistent with an intrinsic instrumental resolution of $194 \mathrm{meV}$ when using the lowest pass energy, $50 \mathrm{eV}$, and the smallest slit $(0.2 \mathrm{~mm}$ curved). Raising the pressure from high vacuum $\left(1.2 \times 10^{-8}\right.$ Torr $)$ to elevated pressures ( 0.4 Torr) did not affect the spectrometer energy resolution, but resulted in an exponential decrease of the intensity of the photoelectron signal. At the maximum attainable pressure in the XPS analysis chamber ( 0.4 Torr), the area of the $\operatorname{Ag~} 3 d_{5 / 2}$ peak decreased to $31 \pm 3 \%$ of the in- tensity of the same peak collected at $1.2 \times 10^{-8}$ Torr. The possibility of acquiring angle-resolved data at different pressures has been demonstrated by analyzing a $\mathrm{Si} / \mathrm{SiO}_{2}$ sample under high vacuum conditions and at elevated pressure. The collected angle-resolved spectra could be effectively used for the accurate determination of the thickness of the native silicon oxide layer (1.3-1.4 nm) under both pressure conditions.

There are several possible ways that the XPS spectrometer could be further developed in the future. In particular, improving the pumping speed of the differentially pumped system (both electrostatic lens system/electron energy analyzer and X-ray source/monochromator) would allow the maximum attainable pressure in the XPS analysis chamber to be increased. As well, focusing the X-rays onto a smaller spot would also permit a further reduction of the size of the apertures mounted in the hemispherical analyzer input lens system and, therefore, the acquisition of XPS spectra at pressures higher than 0.4 Torr.

As already pointed out in Sec. II, this E-XPS system is coupled to a variable temperature UHV scanning probe microscope. Transferring the sample from the E-XPS chamber to the SPM allows the investigation of both sample morphology and nanoscale properties (e.g., adhesion and friction) without any exposure of the sample to air. The coupling of E-XPS and SPM provides unique opportunities to gain a better understanding of surface phenomena in many different fields, such as tribology and catalysis, avoiding the drawbacks associated with exposing the specimen to air (e.g., adsorption of adventitious carbon).

\section{ACKNOWLEDGMENTS}

Financial support of the Swiss National Science Foundation (grant to F.M.) and of the Natural Sciences and Engineering Research Council of Canada (grant to P.E.) is acknowledged. This material is based upon work partially supported by the National Science Foundation under Grant No. DMR-1107642. The acquisition of the XPS instrumentation was partially supported by the U.S. Department of Defense

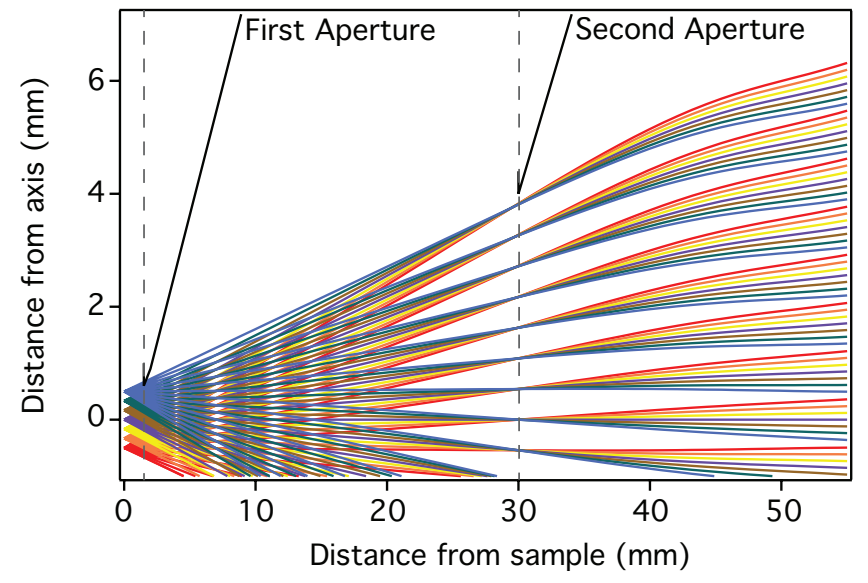

FIG. 10. Electron ray-tracing calculations for the Scienta R3000 HP electrostatic lens system operating in transmission mode. The calculations were performed assuming a pass energy of $100 \mathrm{eV}$ and considering electrons with kinetic energy of $1100 \mathrm{eV}$. 
(a)

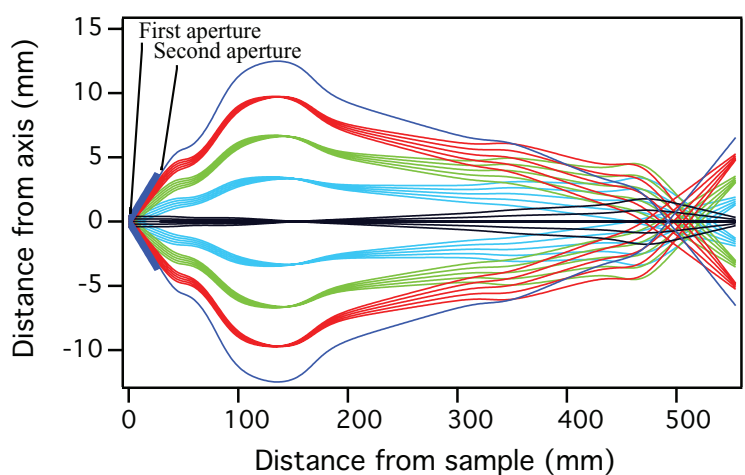

(b)

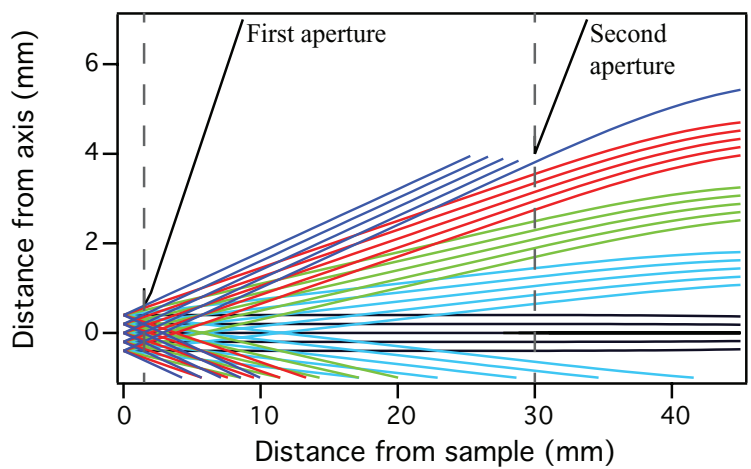

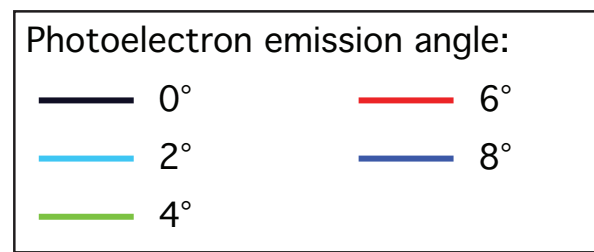

(c)

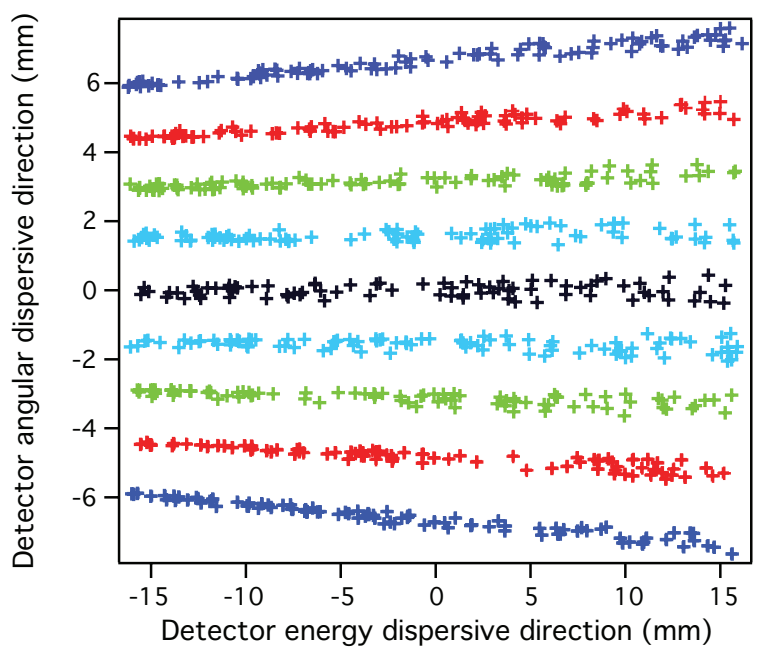

FIG. 11. (a) Electron ray-tracing calculations performed for A10 angular lens mode assuming a pass energy of $100 \mathrm{eV}$ and considering electrons with kinetic energy of $1037.5 \mathrm{eV}$. The simulated region from which photoelectrons are emitted is $0.8 \times 0.8 \mathrm{~mm}^{2}$ and positioned at $\mathrm{x}=0 \mathrm{~mm}, \mathrm{y}=0 \mathrm{~mm}$. Electrons emitted from different positions on the sample surface but with the same emission angle are focused at the same position on the slit at the entrance of the electron energy analyzer. (b) Zoomed view of the electron trajectories shown in (a). The corresponding simulated detector image is reported in (c). The kinetic energy at the center of the $\mathrm{x}$-axis of the detector is $1037.5 \mathrm{eV}$ in the present calculations. The detector window in the energy dispersive plane is $12 \%$ of the pass energy (i.e., $12 \mathrm{eV}$ in the present calculations). The angular distribution of the emitted photoelectrons is dispersed in the direction orthogonal to the energy dispersive plane, i.e., on the y-axis of (c). At the center of this axis, electrons emitted along the electrostatic lens axis are detected. The dispersion is calculated to be $0.9 \mathrm{~mm} /{ }^{\circ}$. The angular resolution is calculated to be less than $0.5^{\circ}$.

DURIP program under Air Force Grant No. FA9550-06-10289. We gratefully acknowledge Dr. M.A. Hamilton for substantially assisting with the installation of the XPS instrumentation. We thank P. Rockett, P. Szczesniak, and G. Mosqueira for machining several structural support elements of the system.

\section{APPENDIX: ELECTRON RAY-TRACING CALCULATIONS}

Electron ray-tracing calculations were performed to investigate the influence of the front two apertures on: (a) the area on the sample surface from which the electrostatic lens collects photoelectrons and (b) the acceptance angle of the lens system.

Figure 10 displays the results of the electron ray-tracing calculations performed assuming a pass energy of $100 \mathrm{eV}$ and considering electrons with kinetic energy of $1100 \mathrm{eV}$. None of the electron trajectories passing through the first aperture are intersected by the second aperture. Therefore, in transmission mode the analyzed area is determined by the first aperture and is about $1.2 \mathrm{~mm}$ in diameter.

Electron ray-tracing calculations were also performed to investigate the influence of the front two apertures on the acceptance angle of the lens system. The results, obtained as- suming a pass energy of $100 \mathrm{eV}$ and considering electrons with kinetic energy of $1037.5 \mathrm{eV}$, are displayed in Figures 11(a) and 11(b). In angle-resolved mode, the acceptance angle of the electrostatic lens is limited to $14^{\circ}$ by the dimension of the second aperture (diameter: $8 \mathrm{~mm}$ ). As mentioned previously, a set of angular lens tables is also available in the analyzer software to limit the angular ranges over which photoelectrons are collected to $6^{\circ}$ (A6 angular mode) and $10^{\circ}$ (A10 angular mode), i.e., below the maximum acceptance angle of the electrostatic lens $\left(14^{\circ}\right)$. The simulated detector image in the case of XPS analysis performed in angular mode is shown in Figure 11(c). The electron kinetic energy is dispersed along one direction of the detector (x-axis in Figure 11(c)), whereas the angular distribution of the emitted photoelectrons is dispersed in the orthogonal direction (y-axis in Figure 11(c)).

${ }^{1}$ B. D. Ratner and D. G. Castner, in Surface Analysis - The Principal Techniques, edited by J. C. Vickerman and I. Gilmore (Wiley, New York, 2009), pp. 47-112.

${ }^{2}$ C. S. Fadley, J. Electron Spectrosc. Relat. Phenom. 178-179, 2-32 (2010). ${ }^{3}$ D. Briggs and J. T. Grant, Surface Analysis by Auger and X-ray Photoelectron Spectroscopy (IM, Chichester, 2003), p. 899.

${ }^{4}$ M. Salmeron and R. Schlögl, Surf. Sci. Rep. 63(4), 169-199 (2008).

${ }^{5}$ H. Bluhm, J. Electron Spectrosc. Relat. Phenom. 177(2-3), 71-84 (2010).

${ }^{6}$ H. Bluhm, M. Hävecker, A. Knop-Gericke, M. Kiskinova, R. Schlögl, and M. Salmeron, MRS Bull. 32(12), 1022-1030 (2007).

${ }^{7}$ D. F. Ogletree, H. Bluhm, G. Lebedev, C. S. Fadley, Z. Hussain, and M. Salmeron, Rev. Sci. Instrum. 73(11), 3872 (2002). 
${ }^{8}$ D. F. Ogletree, H. Bluhm, E. D. Hebenstreit, and M. Salmeron, Nucl Instrum. Methods Phys. Res. A 601(1-2), 151-160 (2009).

${ }^{9}$ H. J. Ruppender, M. Grunze, C. W. Kong, and M. Wilmers, Surf. Interface Anal. 15(4), 245-253 (1990).

${ }^{10}$ M. E. Grass, P. G. Karlsson, F. Aksoy, M. Lundqvist, B. Wannberg, B. S. Mun, Z. Hussain, and Z. Liu, Rev. Sci. Instrum. 81(5), 053106 (2010).

${ }^{11}$ K. Siegbahn, C. Nordling, G. Johansson, J. Hedman, P.-F. Heden, K. Hamrin, U. Gelius, T. Bergmark, L. O. Werme, R. Manne, and Y. Baer, ESCA Applied to Free Molecules (North-Holland, Amsterdam, 1969), p. 201.

${ }^{12}$ F. F. Tao, Chem. Commun. 48(32), 3812-3814 (2012).

${ }^{13}$ A. Jürgensen, N. Esser, and R. Hergenröder, Surf. Interface Anal. 44(8), 1100-1103 (2012).

${ }^{14}$ J. Pantförder, S. Pöllmann, J. F. Zhu, D. Borgmann, R. Denecke, and H.-P. Steinrück, Rev. Sci. Instrum. 76(1), 014102 (2005).

${ }^{15}$ H. Fellner-Feldegg, Ph.D. dissertation, Uppsala Universitet, Uppsala, 1974.

${ }^{16}$ N. Mårtensson, P. Baltzer, P. A. Brühwiler, J. O. Forsell, A. Nilsson, A. Stenborg, and B. Wannberg, J. Electron Spectrosc. Relat. Phenom. 70(2), 117-128 (1994).

${ }^{17}$ B. Wannberg, Nucl. Instrum. Methods Phys. Res. A 601(1), 182-194 (2009).

${ }^{18}$ American Society for Testing and Materials (ASTM) International, Standard Terminology Relating to Surface Analysis (American Society for Testing and Materials International, 2003).

${ }^{19}$ International Organization for Standardization (ISO), Surface Chemical Analysis - X-ray Photoelectron Spectroscopy - Description of Selected Instrumental Performance Parameters (International Organization for Standardization, Geneva, 2000).
${ }^{20}$ J. F. Moulder, W. F. Stickle, P. E. Sobol, and K. D. Bomben, Handbook of $X$-ray Photoelectron Spectroscopy (Perkin-Elmer Corporation, Eden Prairie, MN, 1992), p. 261.

${ }^{21}$ J. J. Olivero and R. L. Longbothum, J. Quant. Spectrosc. Radiat. Transf. 17(2), 233-236 (1977).

${ }^{22}$ J. L. Campbell and T. Papp, At. Data Nucl. Data Tables 77(1), 1-56 (2001).

${ }^{23}$ S. Hüfner, Photoelectron Spectroscopy - Principles and Applications (Springer, Berlin, 2003), p. 662.

${ }^{24}$ S. Mähl, M. Neumann, S. Dieckhoff, V. Schlett, and A. Baalmann, J. Electron Spectrosc. Relat. Phenom. 85(3), 197-203 (1997).

${ }^{25}$ VG Scienta, X-Ray Source Scienta MX650, Uppsala, Sweden, 2006.

${ }^{26}$ F. Aksoy, M. E. Grass, S. H. Joo, N. Jabeen, Y. P. Hong, Z. Hussain, B. S. Mun, and Z. Liu, Nucl. Instrum. Methods Phys. Res. A 645(1), 260-265 (2011).

${ }^{27}$ J. F. Watts and J. Wolstenholme, An Introduction to Surface Analysis by XPS and AES (Wiley, New York, 2003), p. 224.

${ }^{28}$ VG Scienta, Electron Spectrometer SCIENTA R3000, Uppsala, Sweden, 2010.

${ }^{29}$ G. Beamson, D. Briggs, S. F. Davies, I. W. Fletcher, D. T. Clark, J. Howard, U. Gelius, B. Wannberg, and P. Balzer, Surf. Interface Anal. 15(9), 541-549 (1990).

${ }^{30}$ International Organization for Standardization (ISO), Surface Chemical Analysis - X-ray Photoelectron Spectroscopy - Measurement of Silicon Oxide Thickness (International Organization for Standardization, Geneva, 2011).

${ }^{31}$ M. P. Seah and S. J. Spencer, Surf. Interface Anal. 35(6), 515-524 (2003).

${ }^{32}$ A. H. Al-Bayati, K. G. Orrman-Rossiter, J. A. van den Berg, and D. G. Armour, Surf. Sci. 241(1-2), 91-102 (1991). 